\title{
MONITORING CHANGES IN THE COASTAL ENVIRONMENT BASED ON SAR SENTINEL-1 TIME-SERIES
}

\author{
Ramona Pelich $^{\dagger} \quad$ Marco Chini ${ }^{\dagger} \quad$ RenaudHostache ${ }^{\dagger} \quad$ Patrick Matgen ${ }^{\dagger} \quad$ Carlos Lopez-Martinez $z^{\ddagger}$ \\ ramona.pelich@list.lu \\ ${ }^{\dagger}$ Luxembourg Institute of Science and Technology (LIST), ERIN Department, Luxembourg \\ $\ddagger$ Univeristat Politecnica de Catalunya (UPC), Barcelona, Spain
}

\begin{abstract}
This research addresses the use of Sentinel-1 time series with the aim of detecting spatio-temporal changes in the coastal environment. To this end an automatic and unsupervised coastline detection method is proposed. First, we apply a temporal averaging filter that allows encapsulating the temporal variations in coastal areas, e.g. due to tides or vegetation, and at the same time it allows reducing the speckle, without decreasing the spatial resolution of the Synthetic Aperture Radar (SAR) images. Then, based on the distinctive backscattering values of the sea and land classes we employ an iterative hierarchical tiling method in order to accurately characterize the two classes by a bimodal distribution. The latter is then segmented by a thresholding and region-growing procedure to separate the sea and land classes. The proposed method is applied to two different SAR time-series, each one acquired throughout one year. The extracted yearly coastlines are then analyzed in order to identify spatio-temporal changes. Experimental results showcase coastal area changes between occuring 2018 and 2019 and that were caused by the hurricane Michael hitting Northwest Florida in October 2018 .
\end{abstract}

Index Terms - coastal environment, change detection, multi-temporal, segmentation, synthetic aperture radar (SAR).

\section{INTRODUCTION}

Nowadays, approximately $44 \%$ of the world's population lives within 150 kilometers of the coast. The coastal environment is represented by very diverse and dynamic zones across the globe that are used for human settlement, agriculture, trade, industry and amenity [1]. In addition, coastal areas are also represented by natural ecosystems being home to a variety of animal and plant species that provide benefits for our society. The coastal areas are often affected by both natural and man-made changes such as effects of the rising sea level, coastal flooding or pollution, that can lead to changes in both the coastal environment and ecosystem. In this context, it is very important to have access to accurate information about the coastal zones and associated changes overtime. The detection and monitoring of the coastline by means of remote sensing has been demonstrated in many research studies [2], among which Synthetic Aperture Radar (SAR) imagery, being acquired independently of weather conditions and daylight, occupies a privileged place.

Many different algorithms have been developed for the detection/extraction of coastlines from SAR imagery. Pioneer studies in the domain propose to use image-processing techniques coupled with edge detection methods for the shoreline delineation $[3,4]$. Other research studies propose to use wavelets methods [5] or probabilistic models [6] for efficiently delineating the coastline from SAR imagery. Alternatively, polarimetry methods based on the correlation between the co-polarized channels combined with Gaussian-shaped filters [7] or active contour models [8], are used to accurately detect the shoreline from SAR observations. In addition, new SAR-based coastline extraction techniques emerged from recent advances in SAR imaging capabilities, such as those of the Sentinel-1 SAR mission. For instance, the Sentinel1 dual polarization characteristics are employed to propose unsupervised methods based on the correlation between coand cross-polarized channels for delineating the shorelines $[9,10]$. The Sentinel-1 extracted coastline is also used for addressing temporal changes between images acquired at different time instants as presented in [10].

In this paper, we introduce an unsupervised and automatic SAR method for a precise detection of the coastline extent. The method encapsulates all its periodical variations, e.g. due to tides or vegetation, by making use of Sentinel-1 SAR timeseries. The proposed method is then applied to two different time series each one acquired over two different years. The aim of this study is to compare the estimated yearly coastlines in order to identify long-term spatial changes affecting the coastal environment.

\section{METHODOLOGY}

In this study we define the coastline as a geographical variable/map that encompasses all the constant dynamical vari- 


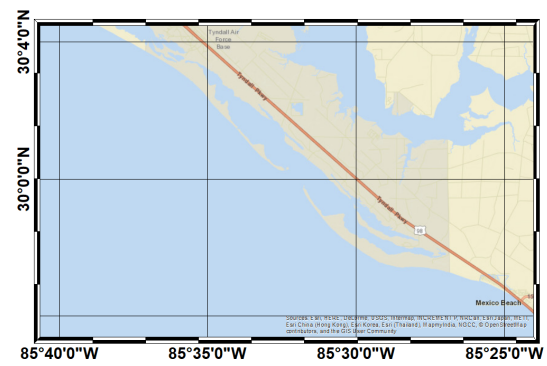

(a)

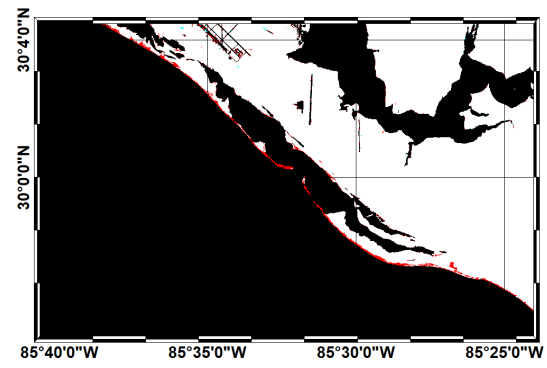

(d)

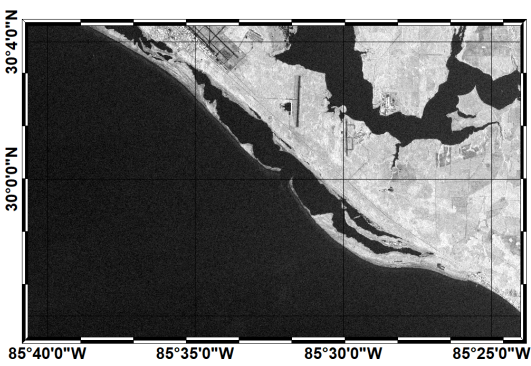

(b)

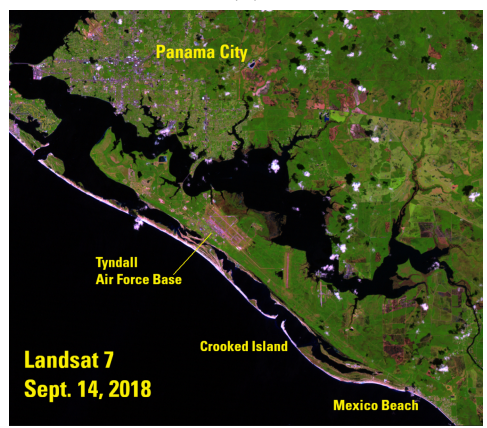

(e)

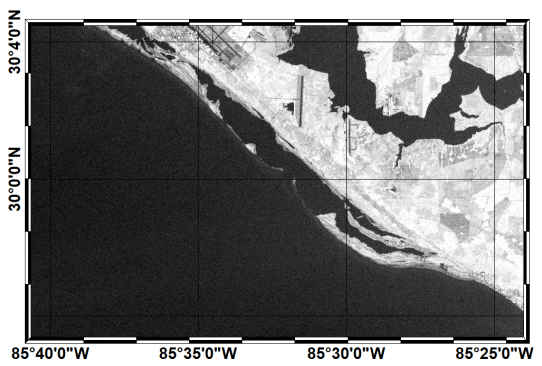

(c)

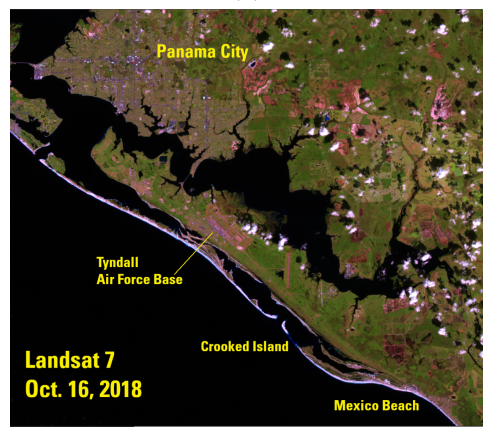

(f)

Fig. 1: Case study 1: Area of interest (a); Mean of multi-temporal Sentinel-1 intensity images acquired over 2018 (b) and 2019 (c); RGB composite of the SAR derived coastline: $\mathrm{R}=2018, \mathrm{G}=\mathrm{B}=2019$ (d); Illustration of NOAA images acquired before (e) and after (f) Hurricane Michael, source: https:/www.usgs.gov/media/images/and-after-photos-show-hurricane-michaelsdestructive-power-2 (last accessed in January 2020).

ations of the coastal zones within a limited period of time, e.g. one year. We suppose that in a SAR time-series spanning one year the most relevant and representative dynamics influencing the location of the coastline will be considered. We consider two main types of dynamical variations: (i) classical coastal changes due for example to tides, waves or currents that occur periodically (e.g. daily) and (ii) complex dynamics generated by vegetated coastal areas (e.g. deltas, wetlands), erosion/sedimentation processes or human interventions (e.g. dikes, walls) of a longer time scale evolution ranging from weeks to months. In this context, the Sentinel1 Copernicus mission providing access to large SAR image datasets available due to its high orbit revisit time (6 days), is particularly well-suited for computing the coastline from SAR time-series. Based on the Sentinel-1 image stacks we propose a coastline detection technique composed of the following steps:

- Multi-temporal speckle filtering: temporal averaging is a common filtering solution that allows to filter the speckle without decreasing the spatial resolution of the SAR images [11]. In SAR imagery the water class is generally represented by very low backscattering values. Averaging SAR time-series of a sufficiently long time period permits reducing the speckle of the water class, thereby making it separable from the land class.

- Selection of bimodal tiles along the coastline: automatic selection of tiles with a balanced number of sea and land pixels and an identifiable bimodal distribution as proposed in the HSBA method [12].

- Bimodal distribution classification: estimation of the bimodal distribution parameters from the selected bimodal tiles followed by a thresholding and region growing procedure.

- Iterative application of the above steps to different yearly SAR datasets in order to identify long term spatio-temporal changes of the coastline.

\section{EXPERIMENTAL RESULTS}

Across the globe, many countries located in coastal areas have to deal with high risks of damage caused by natural disasters. Numerous devastating water-related disasters have been hitting coastal regions all over the world. For example, in 2018 Hurricane Michael hit Florida's Panhandle coast, causing major flood damage to coastal infrastructures and amenities. For instance, strips of shoreline disappeared beneath the water and large road sections were completely destroyed.

The SAR dataset employed in this study is composed of Sentinel-1 Ground Range Detected (GRD) images in the interferometric wide swath (IW) mode, with a spatial resolution $\mathrm{rg} \times$ az of approximately $5 \times 20 \mathrm{~m}$. The coastline extraction is performed independently from two stacks of 10 Sentinel-1 images from the same orbital track, acquired throughout different time periods: the first one is comprised of images ac- 


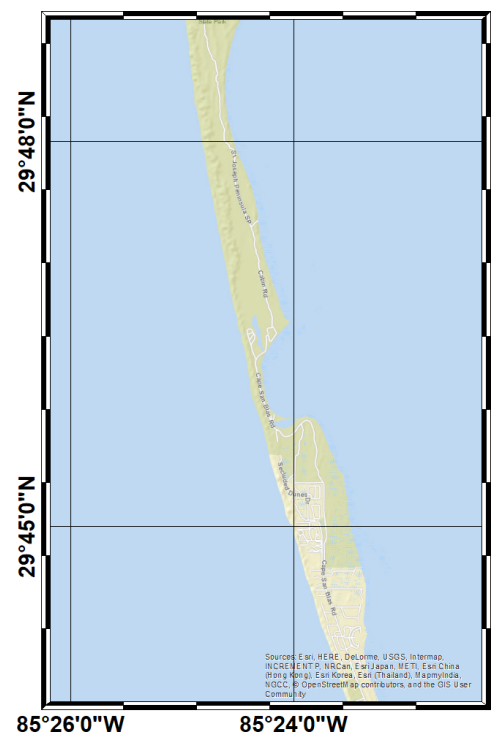

(a)

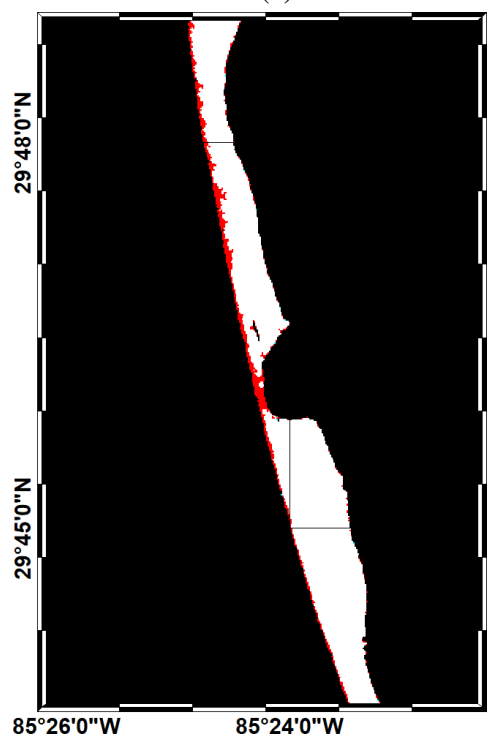

(d)

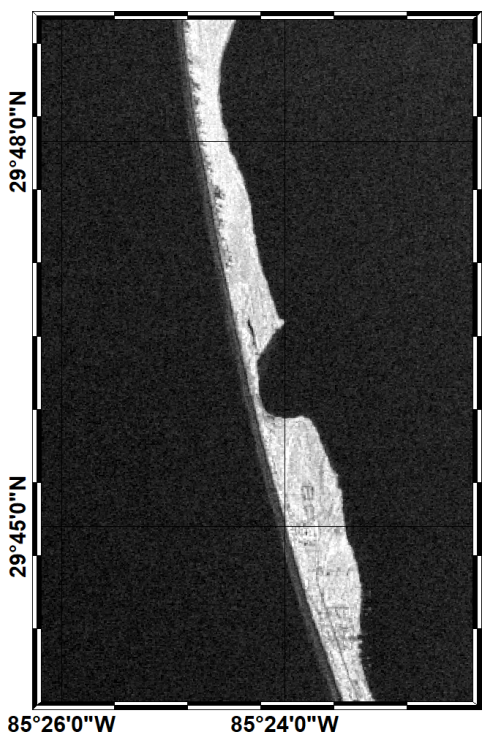

(b)

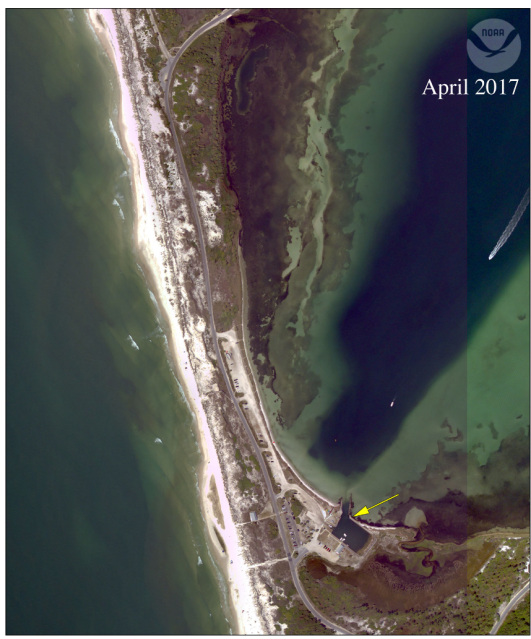

(e)

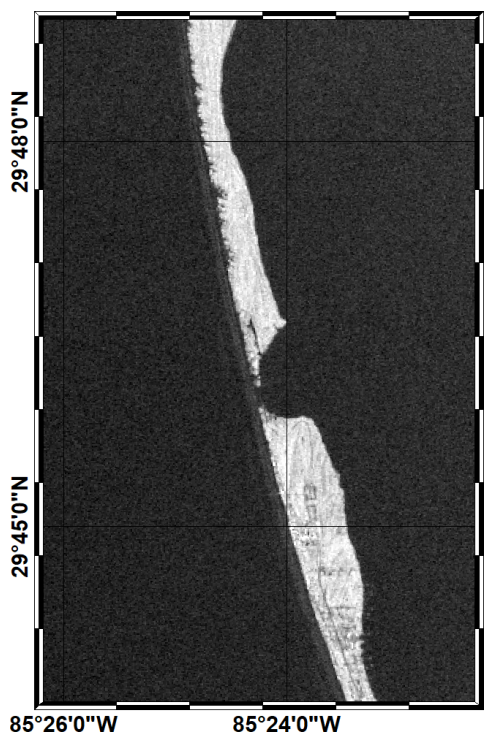

(c)

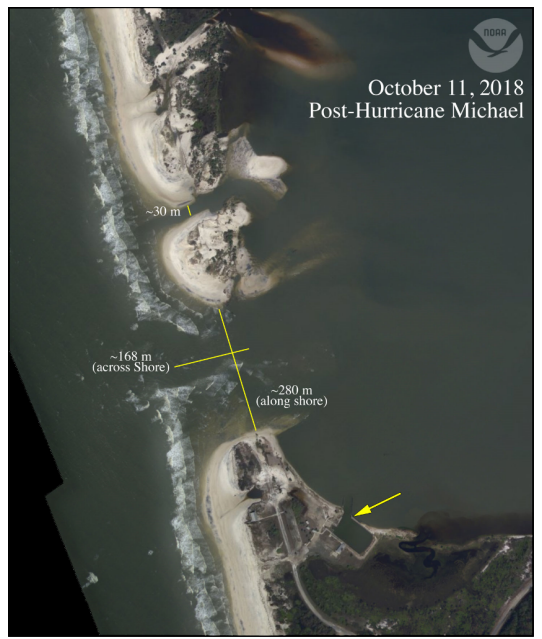

(f)

Fig. 2: Case study 2: Area of interest (a); Mean of multi-temporal Sentinel-1 intensity images acquired over 2018 (b) and 2019 (c); RGB composite of the SAR derived coastline: $\mathrm{R}=2018, \mathrm{G}=\mathrm{B}=2019$ (d); Illustration of Landsat images acquired before (e) and after (f) Hurricane Michael: https://www.usgs.gov/media/before-after/hurricane-michael-batters-florida-panhandle (last accessed in January 2020)

quired in 2018 before the Hurricane Michael (October 2018) and the second one is comprised of images acquired throughout 2019 after Hurricane Michael. In the following we will address two different case studies from Northwest Florida that showcases coastal changes caused by Hurricane Michael.

Figure 1 showcases the coastal area located south of Panama City, Florida that has been affected by Hurricane Michael causing widespread damage in the area. From the Sentinel-1 multi-temporal imagery acquired in 2018 and 2019 shown in Figures 1 (b) and (c) respectively, we can notice that there are differences between coastline, especially for the shoreline strips located along Crooked Island. By analysing the result of the 2018 and 2019 coastline comparison presented in Figure 1 (d) we can observe that shoreline strips have been broken and disappeared beneath the water (Crooked Island) while beaches, e.g. Mexico beach, have been partially eroded. Optical NOAA images acquired just before and after Hurricane Michael confirm similar changes of the coastline in this area as illustrated in 1 (e) and (f).

Figure 2 showcases the coastal area located near Cape San 
Blas that is part of a peninsula in Gulf County, Florida. We can notice from the SAR imagery that also in case there are differences between the 2018 and 2019 shorelines. The extracted SAR coastlines given in Figure 2 (c) indicate that a narrow part of the shoreline has been completely eroded and submerged and beaches located in the western of the peninsula have been eroded. From Landsat imagery acquired before and after Hurricane Michael illustrated in Figures 1 (e) and (f) we notice the erosion of the narrow shoreline that lead to destruction of the road that was providing access to T.H. Stone Memorial St. Joseph Peninsula State Park.

By analysing and comparing the yearly coastline we are able to identify long-term spatio-temporal changes affecting the coastal environment.

\section{REFERENCES}

[1] UN Atlas of the Oceans, "Human Settlements on the Coast," http://www.oceansatlas.org/subtopic/en/c/114/.

[2] Rudiger Gens, "Remote sensing of coastlines: detection, extraction and monitoring," International Journal of Remote Sensing, vol. 31, pp. 1819-1836, 2010.

[3] Jong-Sen Lee and Igor Nikolaevich Jurkevich, "Unsupervised Coastline Detection And Tracing In SAR Images," IEEE TGRS, vol. 24, pp. 662-668, 1990.

[4] David C. Mason and Ian J. Davenport, "Accurate and efficient determination of the shoreline in ERS-1 SAR images," IEEE TGRS, vol. 34, pp. 1243-1253, 1996.

[5] Marivi Tello, Carlos López-Martínez, Jordi J. Mallorquí, and Philippe Salembier, "Edge Enhancement Algorithm Based on the Wavelet Transform for Automatic Edge Detection in SAR Images," IEEE TGRS, vol. 49, pp. 222-235, 2011.

[6] Fabio Baselice and Giampaolo Ferraioli, "Unsupervised Coastal Line Extraction From SAR Images," IEEE GRSL, vol. 10, pp. 1350-1354, 2013.

[7] Ferdinando Nunziata, Maurizio Migliaccio, Xiaofeng Li, and Xianwen Ding, "Coastline Extraction Using Dual-Polarimetric COSMO-SkyMed PingPong Mode SAR Data," IEEE GRSL, vol. 11, pp. 104-108, 2014.

[8] Chun Liu, Yingying Xiao, and Jian Yang, "A Coastline Detection Method in Polarimetric SAR Images Mixing the RegionBased and Edge-Based Active Contour Models,' IEEE TGRS, vol. 55, pp. 3735-3747, 2017.

[9] Ferdinando Nunziata, Andrea Buono, Maurizio Migliaccio, and Guido Benassai, "Dual-Polarimetric C- and X-Band SAR Data for Coastline Extraction," IEEE JSTARS, vol. 9, pp. 4921-4928, 2016.

[10] Dongyang Ao, O. Dumitru, Gottfried Schwarz, and Mihai Datcu, "Coastline detection with time series of SAR images," in Proc. SPIE 10422, Remote Sensing of the Ocean, Sea Ice, Coastal Waters, and Large Water Regions, 2017.

[11] Marco Chini, Ramona Pelich, Renaud Hostache, Patrick Matgen, and Carlos López-Martínez, "Towards a 20 m Global Building Map from Sentinel-1 SAR Data," Remote Sensing, vol. 10, pp. 1833, 2018.
[12] Marco Chini, Renaud Hostache, Laura Giustarini, and Patrick Matgen, "A hierarchical split-based approach for parametric thresholding of sar images: Flood inundation as a test case," IEEE TGRS, vol. 55, pp. 6975-6988, 2017. 